\begin{tabular}{|c|l|}
\hline Title & $\begin{array}{l}\text { Programmable nano-switch array using SiN GaA sinterface traps on a GaA s nanowire network for reconfigurable BDD } \\
\text { logic circuits }\end{array}$ \\
\hline Author(s) & Shiratori, Y uta; Miura, Kensuke; Kasai, Seiya \\
\hline Citation & $\begin{array}{l}\text { Microelectronic Engineering, 88(8), 2755-2758 } \\
\text { https://doi.org/10.1016/.mee.2010.12.007 }\end{array}$ \\
\hline Issue Date & 2011-08 \\
\hline Doc URL & http://hdl.handle.net/2115/47136 \\
\hline Type & article (author version) \\
\hline File Information & ME88_8_2755-2758.pdf \\
\hline
\end{tabular}

Instructions for use 


\title{
Programmable Nano-Switch Array Using SiN/GaAs Interface Traps on a GaAs Nanowire Network for Reconfigurable BDD Logic circuits
}

\author{
Yuta Shiratori $^{1 *}$, Kensuke Miura $^{1}$, and Seiya Kasai ${ }^{1,2}$ \\ ${ }^{1}$ Research Center for Integrated Quantum Electronics, and Graduate School of \\ Information Science and Technology, Hokkaido University, North 13, West 8, Sapporo \\ 060-8628, Japan \\ ${ }^{2}$ PRESTO, Japan Science and Technology Agency, 4-1-8 Honcho, Kawaguchi, Saitama \\ 332-0012, Japan
}

\begin{abstract}
Programmable nano-switch arrays on GaAs-based nanowire networks are investigated for a reconfigurable binary-decision-diagram (BDD) logic circuit. A programmable switch was simply realized by inserting a $\operatorname{SiN}_{x}$ thin layer between a metal gate and a nanowire. Fabricated switches were characterized in terms of hysteresis curve, program time dependences of off-state retention time, and on-state current. $\mathrm{HCl}$ treatment on $\mathrm{SiN}_{x}$ prior to metal gate formation was found to remarkably improve the switching characteristics. We experimentally demonstrated correct and stable operation of a four-input reconfigurable BDD circuit integrating the switch array with $\mathrm{HCl}$ treatment.

*E-mail address: siratori @ rciqe.hokudai.ac.jp
\end{abstract}




\section{Introduction}

Semiconductor nanowire is a promising candidate for the transistor channel material in logic large-scale integrated circuits (LSIs) beyond 10-nm technology [1-2]. Bottom up formation technique is expected for its mass production because small fine nanowires can be produced in a simple way with low cost [2]. On the other hand, problems are in the flexibility and accuracy in position control, which are strongly necessary in the current LSI physical architecture. Thus alternative circuit architectures utilizing nanowire and related structures have been investigated [3,4]. We have proposed and developed a binary-decision-diagram (BDD)-based logic circuit, which is implemented on a regular nanowire network structure [5-9]. Furthermore, we recently proposed and demonstrated a reconfigurable BDD logic circuit utilizing a programmable switch array [10]. This circuit can change its logic function dynamically by external programming signals without changing the physical network configuration. Therefore various logic functions can be implemented on a nanowire network structure. The performance of the circuit such as reconfiguration speed and stability depends directly on the performance of the switches. The purpose of this paper is to investigate the operation of the programmable nano-switches formed on GaAs-based nanowires in detail. Their effect on the circuit operation is confirmed by fabrication and characterization of four-input reconfigurable BDD circuits.

\section{Concept of reconfigurable BDD circuits}

Schematic of the reconfigurable BDD logic circuit on GaAs nanowire network is shown in Fig. 1. The physical nanowire network represents a universal graph of Boolean functions using Shannon's expression [10]. Programmable nano-switch array is integrated in leaves of the graph and connected to a common terminal. Circuit reconfiguration is made by connecting or disconnecting the leaves and the terminal by 
switching nanowire conductance in the switch array. In this study, the switch was realized by inserting a $\mathrm{SiN}_{\mathrm{x}}$ thin layer between a metal gate electrode and a GaAs nanowire. In this system, electron traps are known to be formed in $\mathrm{SiN}_{\mathrm{x}} / \mathrm{GaAs}$ interface [11]. The conductance in the nanowire is switched on and off by charging or discharging the $\mathrm{SiN}_{\mathrm{x}} / \mathrm{GaAs}$ interface traps. On and off states are programmed with negative and positive gate voltages, respectively. After programming, a conduction state is preserved even when the gate voltage is turned off. This switch has a simple structure and is realized by only adding $\mathrm{SiN}_{\mathrm{x}}$ deposition process to circuit fabrication process.

\section{Experimental}

Nanowires were formed by EB lithography and wet chemical etching an (100) AlGaAs/GaAs modulation doped heterostructure wafer. The wire direction was $\langle 0-11\rangle$ and the sidewall facet was (111)B. For programmable nano-switches, $\operatorname{SiN}_{\mathrm{x}}(\mathrm{x}=1.2)$ insulating layer was deposited by electron cyclotron resonance chemical vapor deposition (ECR-CVD) at a substrate temperature $260{ }^{\circ} \mathrm{C} . \mathrm{SiN}_{\mathrm{x}}$ thickness was about 10 nm. For good interface formation in the device fabrication, the surface treatment is important to remove surface chemical contamination. Prior to the gate metal formation, $\mathrm{SiN}_{x}$ surface was treated by $5 \% \mathrm{HCl}$ solution. The device without the $\mathrm{HCl}$ treatment was also fabricated for comparison. A PtPd gate electrode was formed by sputtering. Four-input reconfigurable circuits were also fabricated on hexagonal nanowire networks with the same process. In the circuit fabrication, PtPd Schottky wrap gates (WPGs) for path swtiches were formed prior to deposition of $\mathrm{SiN}_{x}$ layer.

\section{Results and discussion}

Figure 2 shows hysteresis characteristics of fabricated switches with and without the $\mathrm{HCl}$ treatment. The wire width and the gate length were $610 \mathrm{~nm}$ and $480 \mathrm{~nm}$, 
respectively. The measurement was carried out at room temperature (RT). The initial threshold voltages, $V_{\text {th0 } 0}$, for switches with and without $\mathrm{HCl}$ treatment were $-0.6 \mathrm{~V}$ and $-0.9 \mathrm{~V}$, respectively. Fabricated switches showed clockwise hysteresis. This result confirmed that electrons traps were formed by inserting the $\mathrm{SiN}_{x}$ layer in the gate. Both on and off states of the nanowire conductance at $V_{\mathrm{G}}=0 \mathrm{~V}$ were successfully realized. The amount of hysteresis depended on the surface treatment. Large hysteresis of $2 \mathrm{~V}$ was obtained in the switch with $\mathrm{HCl}$ treatment. Estimated trapped density from the hysteresis curves in the devices with and without the treatment was $2.4 \times 10^{12} \mathrm{~cm}^{-2}$ and $1.4 \times 10^{12} \mathrm{~cm}^{-2}$, respectively.

Figure 3(a) shows the program-time dependence of the off-state retention time, $\tau_{\mathrm{R}}$. It was defined as a time period in which $V_{\text {th }}$ was larger than $0 \mathrm{~V}$ after off-state programming. Programming voltage, $V_{\mathrm{P}}$, was $2 \mathrm{~V}$. Here we focused on the off state because the nanowires always appeared the on-state in initial and the stability of the circuit operation was determined by the off state. $\tau_{\mathrm{R}}$ increased rapidly when the programming time increased from 0 to $10 \mathrm{~ns}$. When the programming was longer than $10 \mathrm{~ns}$, it became almost constant. The increase of $\tau_{\mathrm{R}}$ in the short programming time was due to RC delay by a gate resistance of $100 \Omega$ and a parasitic cable capacitance of 100 pF. $\tau_{\mathrm{R}}$ of the switch with $\mathrm{HCl}$ treatment was more than tenfold of that without the treatment. Considering that the present system was a short channel high-electron-mobility transistor (HEMT) with $\mathrm{SiN}_{x} / \mathrm{GaAs}$ interface traps, $\tau_{\mathrm{R}}$ is theoretically given by $[12,13]$

$$
\tau_{R}=\tau_{e} \ln \left(-\frac{q n_{T}}{C_{A l G a A s} V_{t h 0}}\right)=\tau_{e} \ln \left(-\frac{\alpha V_{P}}{V_{t h 0}}\right)
$$

where $\tau_{\mathrm{e}}$ is the emission time constant of interface traps, $q$ is the elemental charge, $n_{\mathrm{T}}$ is 
the trapped electron density. $n_{\mathrm{T}}$ is empirically expressed as $\alpha C_{\mathrm{AlGaAs}} V_{\mathrm{P}} / q$, where $C_{\mathrm{AlGaAs}}$ is the capacitance of a $\mathrm{AlGaAs}$ layer. $\alpha$ is $V_{\mathrm{SiN}} /\left(V_{\mathrm{AlGaAs}}+V_{\mathrm{SiN}}\right)$ where $V_{\mathrm{SiN}}$ and $V_{\mathrm{AlGaAs}}$ are voltage drops in the $\mathrm{SiN}_{\mathrm{x}}$ and the $\mathrm{AlGaAs}$ layers, respectively. Calculated off-state retention time using Eq. (1) with $\alpha$ of 0.8 and threshold voltages in Fig. 2 were plotted in Fig. 3(a). The experimental data agreed with the theoretical values. The large difference of the retention time is understood by the difference of emission time constant, $\tau_{\mathrm{e}}$.

$\tau_{\mathrm{e}}$ increases when the trap level became deep. Trap energy from a GaAs conduction band edge, $E_{\mathrm{T}}$, was evaluated from Arrhenius plot of $\tau_{\mathrm{e}} . E_{\mathrm{T}}$ in the devices with and without $\mathrm{HCl}$ treatment was $280 \mathrm{meV}$ and $370 \mathrm{meV}$, respectively. Both energies corresponded to the reported $\mathrm{SiN}_{\mathrm{x}} / \mathrm{GaAs}$ interface trap levels [11]. On the other hand, it has not been clarified that the $\mathrm{HCl}$ treatment resulted in deeper energy traps with high density. Its possible role is to supply $\mathrm{H}^{+}$ions. Abrupt slope in the transfer curve as compared to that without the treatment in Fig. 2 indicated the decrease of the continuous interface states. Assuming the diffusion of $\mathrm{H}^{+}$, this result could be understood by the termination of dangling bond by $\mathrm{H}^{+}$. In addition, $\mathrm{H}^{+}$in the $\mathrm{SiN}_{x}$ is known to create discrete electron traps [14], which might be deep and high density. One possible way to confirm that $\mathrm{H}^{+}$plays a dominant role is using other surface treatments including hydrogen. For example, $\mathrm{H}_{2} \underline{\mathrm{SO}}_{4} \underline{\text { solution or }}_{2} \underline{\mathrm{H}}_{2}$ annealing should affect similarly as $\mathrm{HCl}$ treatment. However, further investigation is necessary to confirm that such diffusion of $\mathrm{H}^{+}$occurs.

Short programming time is desirable for switching the conductance in the dynamic reconfiguration of circuits. Figure 3(b) shows the on-state current in the switch as a function of the programming time. $V_{\mathrm{P}}$ was $-2 \mathrm{~V}$. The current was normalized with saturated current obtained at $10 \mathrm{sec}$ after the programming. Minimum on-state programming time, $\tau_{\text {Ponmin }}$ was defined as the time where the normalized current 
reached unity. Evaluated $\tau_{\text {Ponmin }}$ of were $3 \times 10^{-4} \mathrm{sec}$, almost in the same for two devices. The on-state programming took longer time than the off-state one. This is simply because the on-state is realized by detrapping of electrons and this process takes longer time than capturing due to the existence of the energy barrier of $E_{\mathrm{T}}$. It was found that $\tau_{\text {Ponmin }}$ could be decreased by increasing $V_{\mathrm{p}}$. Large negative programming voltage causes high field in the system and enhances the carrier emission by the field-assisted tunneling [15]. The details will be reported elsewhere.

Effects of the performance of the switches in integrated circuit level were investigated experimentally. Figure 4 shows a SEM image of a fabricated four-input reconfigurable BDD circuit on a hexagonal nanowire network. 15 path switch and 16 programmable switches were integrated in this circuit. The circuit area was $38 \times 58 \mu \mathrm{m}^{2}$. All of four-input Boolean logics $\left(2^{n}=65526\right.$ functions, where $n$ is the number of programmable switches) can be operated with only this circuit.

Figure 5 shows measured input-output waveforms of fabricated reconfigurable circuits integrating switch arrays without and with the $\mathrm{HCl}$ treatment. As an example, circuits were programmed to operate a NOR function using program voltage of $\pm 4 \mathrm{~V}$ for $1 \mathrm{~s}$ and $\pm 2 \mathrm{~V}$ for $10 \mathrm{~ms}$ without and with the $\mathrm{HCl}$ treatment, respectively. Input signal frequency was $100 \mathrm{~Hz}$. Electrons were injected from the root and the current in the terminal was measured as output. Both fabricated circuits operated correctly at RT. In case of integrating a switch array without $\mathrm{HCl}$ treatment, the base line of the output gradually increased with time as shown in Fig. 5(a), even though high voltage programming was made for long time. Such unstable operation was caused by incomplete off-state in the switch array due to short retention time. On the other hand, as expected from the data in Fig. 3(a), stable operation of the switches with $\mathrm{HCl}$ treatment results in the stable operation of the circuit. Stable operation could maintain for $2 \mathrm{sec}$, which corresponded to the off-state retention time in Fig. 3(a). Observed 
behaviors indicated that the retention time and the switching speed of the conduction state in the switch array directly influenced the stability and reconfiguration speed of the circuit. From the data in Fig. 3(b), circuit reconfiguration within $0.25 \mathrm{msec}$ is possible when $V_{\mathrm{P}}= \pm 2 \mathrm{~V}$ and it will be further reduced by increasing $V_{\mathrm{p}}$. If the operation frequency is increased, the programming time becomes short and conductance switching becomes insufficient. From the data in Fig. 3, the programmable switch operation degraded when $\mathrm{f}>3 \mathrm{kHz}$. On the other hand, the obtained programming time is similar between devices with and without $\mathrm{HCl}$ treatment. Therefore it is thought that the $\mathrm{HCl}$ treatment does not degrade the operation as compared with that without treatment even though frequency is increased.

A possible approach to realize long stable circuit operation is interface engineering in the switch to control band bending in the SiN/GaAs interface to relax build-in field so as to suppress carrier emission from the trap at zero gate bias.

\section{Conclusions}

Programmable nano-switch arrays on GaAs-based nanowire networks were investigated for a reconfigurable binary-decision-diagram (BDD) logic circuit. Programmable switches were fabricated by inserting a $\mathrm{SiN}_{\mathrm{x}}$ thin layer between a metal gate and a nanowire. The sample in which $\mathrm{HCl}$ treatment was made on the $\mathrm{SiN}_{x}$ prior to metal gate formation remarkably improved hysteresis curve, program time dependences of off-state retention time, and on-state current. We successfully fabricated a four-input reconfigurable BDD circuit integrating the switch array and demonstrated correct and stable operation of the circuit with $\mathrm{HCl}$ treatment process.

\section{Acknowledgements}

The authors thank Professors T. Fukui and T. Hashizume for their continuous 
support. This work was partly supported by a Grant-in-Aid for JSPS Research Fellows and Specially Promoted Research Project (No. 18002003) from MEXT, Japan. 


\section{References}

[1] International Technology Roadmap for Semiconductors 2007 edition, Emerging Research Materials [http://www.itrs.net/Links/2007ITRS/Home2007.htm].

[2] International Technology Roadmap for Semiconductors 2007 edition, Emerging Research Devices [http://www.itrs.net/Links/2007ITRS/Home2007.htm].

[3] S. Eachempati, V. Saripalli, N. Vijaykrishnan, and S. Datta: Proc. IEEE/ACM Int. Symp. Nanoscale Architectures 2008, p. 61.

[4] G. Snider, P. Kuekes and R. S. Williams: Nanotechnology, 15 (2004) 881-891.

[5] S. Kasai and H. Hasegawa: IEEE Electron Device Lett., 23 (2002) 446-448.

[6] H. Hasegawa and S. Kasai: Physica E, 11 (2001) 149-154.

[7] S. Kasai, M. Yumoto, H. Hasegawa: Solid-State Electron., 47 (2003) 199-204.

[8] S. Kasai, T. Nakamura, Y. Shiratori, T. Tamura: J. Computational and Theoretical Nanoscience, 4 (2007) 1120-1132.

[9] H.-Q. Zhao, S. Kasai, Y. Shiratori, T. Hashizume: Nanotechnology, 20 (2009) 245203.

[10] Y. Shiratori, K. Miura, R. Jia, N.-J. Wu, and S. Kasai: Appl. Phys. Express, 3 (2010) 025002.

[11] I. Thurzo and J. Ivanco: Appl. Surf. Sci., 108 (1997) 187-196.

[12] S. M. Sze and Kwok K. Ng: The Third Edition of the standard textbook and reference in the field of semiconductor devices (WILEY-INTERSCIENCE, Hoboken, 2006) p. 406.

[13] H. Sasaki, K. Hayashi, T. Fujioka, K. Mizuguchi, T. Osaki, K. Sugahara, R. Konishi, H. Kasada, and K. Ando: Appl. Surf. Sci., 117/118 (1997) 729-734.

[14] Y. C. Park, W. B. Jackson, D. L. Smith, and N. M. Johnson: J. Appl. Phys., 74 (1993) 381-386 
[15] T.-E. Chang, C. Huang, and T. Wang: IEEE Trans. Electron Devices, 42 (1995) $738-748$ 


\section{Figure captions}

Figure 1 Schematics of a reconfigurable BDD logic circuit and a programmable nano-switch array on a $\mathrm{AlGaAs} / \mathrm{GaAs}$ hexagonal nanowire network.

Figure 2 Hysteresis characteristics of fabricated programmable switches.

Figure 3 Program time dependences of (a) Retention time after off-programming which $V_{\mathrm{P}}=2 \mathrm{~V}$, (b) current recovery ratio after on-programming which $V_{\mathrm{P}}=-2 \mathrm{~V}$. The wire width and the gate length were $480 \mathrm{~nm}$, and $600 \mathrm{~nm}$, respectively.

Figure 4 SEM image of a fabricated four-input reconfigurable BDD circuit.

Figure 5 Input-output waveforms of fabricated circuits programmed to operate NOR.

(a) without $\mathrm{HCl}$ treatment and (b) with $\mathrm{HCl}$ treatment. 
Fig.1

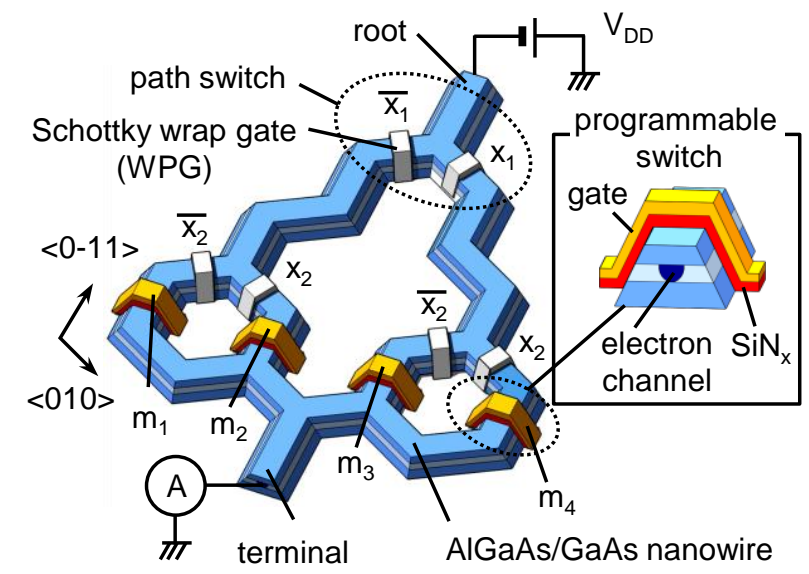




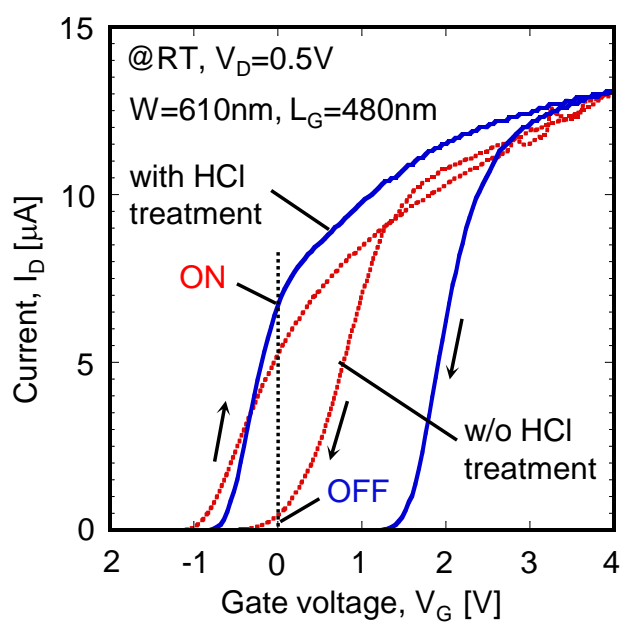




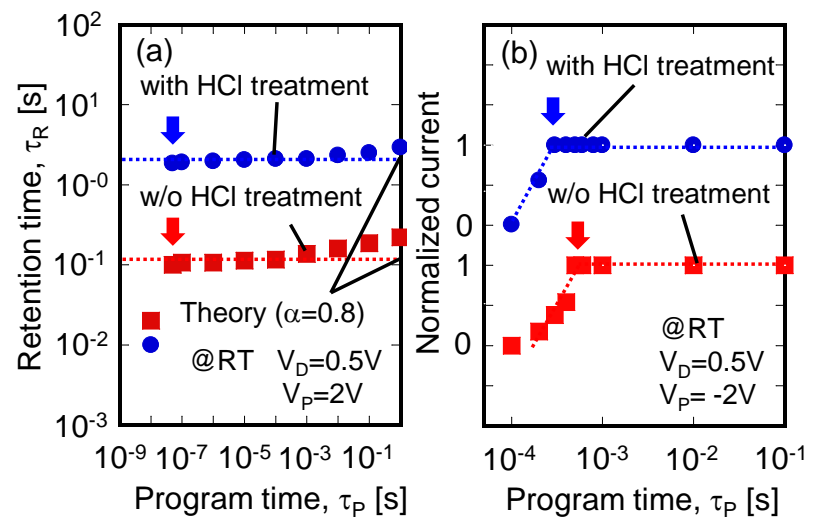




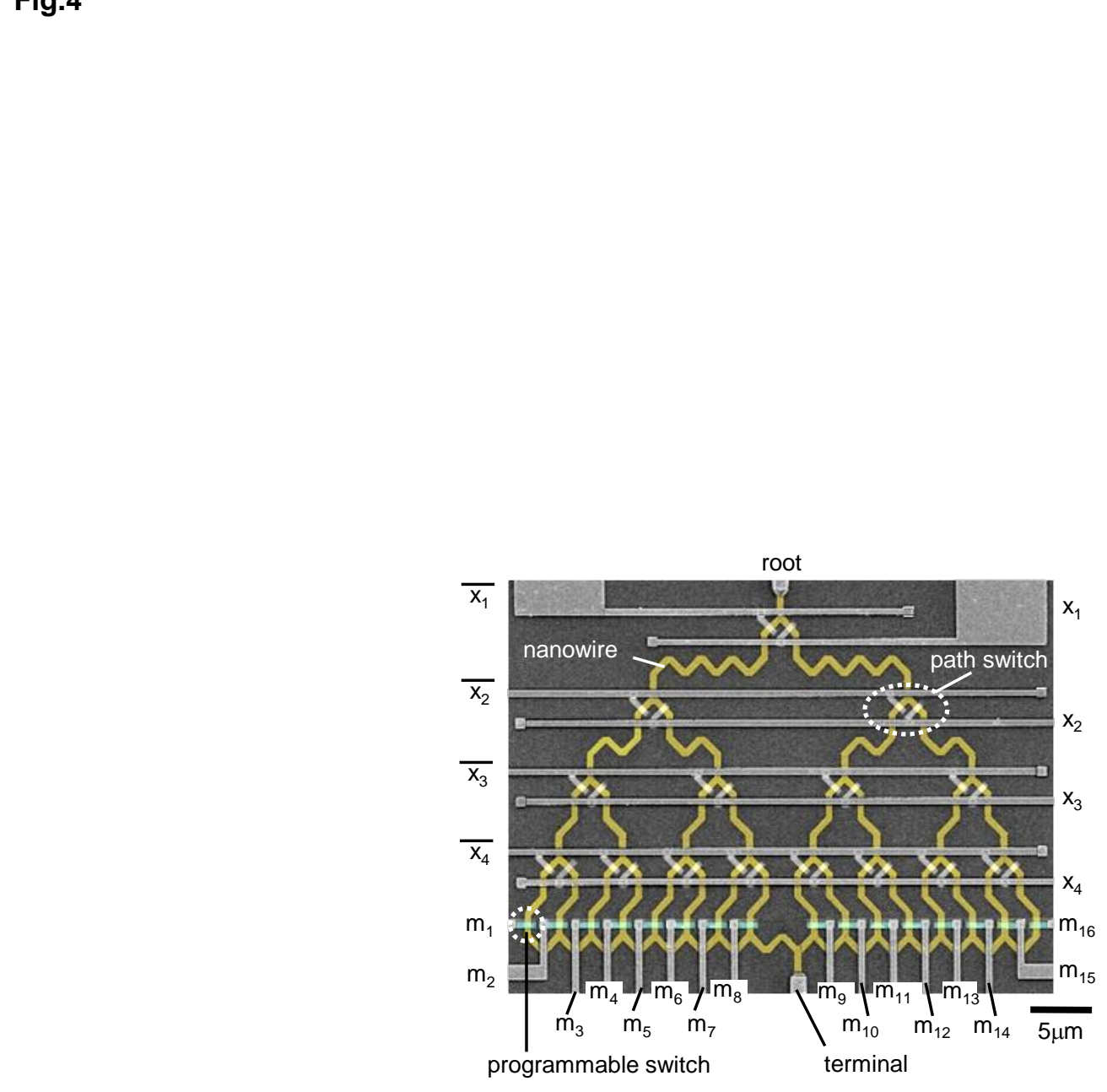

Fig.4

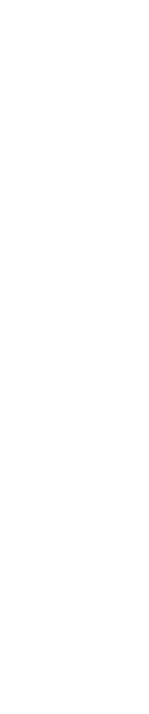

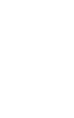
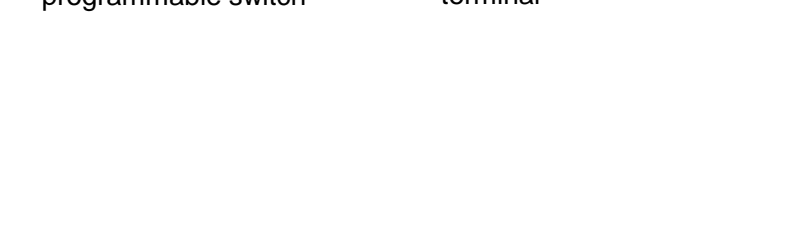
Fig.5

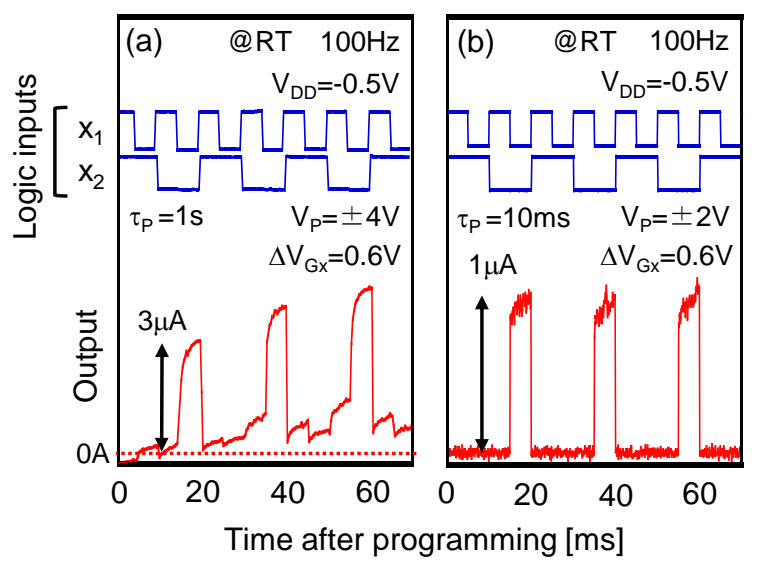

